\title{
The Rho GTPase RhoB regulates cadherin expression and epithelial cell-cell interaction
}

\author{
Francisco M Vega ${ }^{1,2^{*}}$, Mairian Thomas ${ }^{1}$, Nicolas Reymond ${ }^{1}$ and Anne J Ridley ${ }^{1 *}$
}

\begin{abstract}
Background: The Rho GTPase RhoB has been proposed to be a tumor suppressor in cancer and is downregulated in various tumors including prostate. RhoB has different effects on cell migration depending on the cell type and conditions, but the molecular basis for this variability is unclear. RhoB regulates trafficking of membrane receptors and integrins. We have previously shown that RhoB depletion alters focal adhesion dynamics and reduces surface levels of $\beta 1$ integrin in PC3 prostate cancer cells, correlating with increased migration speed.

Results: Here we show that RhoB depletion reduces cell-cell adhesion and downregulates E-cadherin levels as well as increasing internalized E-cadherin in DU145 prostate cancer cells. This is accompanied by increased migration speed. RhoB localizes to cell-cell junctions together with E-cadherin in DU145 cells. RhoB depletion also reduces $\mathrm{N}$-cadherin levels in PC3 cells, which do not express E-cadherin.

Conclusions: These results indicate that RhoB alters migration of cells with cell-cell adhesions by regulating cadherin levels. We propose that the relative contribution of integrins and cadherins to cell migration underlies the variable involvement for RhoB in this process and that the downregulation of RhoB in some epithelial cancers could contribute to the weakening of epithelial cell-cell junction during tumor progression.
\end{abstract}

Keywords: Rho GTPases, RhoB, Cadherins, Adherens junctions, Prostate cancer

\section{Background}

The Rho family of small GTPases are signalling molecules that regulate many cellular processes including cytoskeletal dynamics, cell motility, cell adhesion, cell division and transcription. They thereby contribute to wound healing, inflammation and cancer progression [1]. Most Rho family GTPases cycle between an active GTP-bound state and an inactive GDP-bound state. Their activation is controlled by guanine nucleotide exchange factors (GEFs) and GTPase activating proteins (GAPs), which activate or inactivate them respectively. In their active GTP-bound form, Rho GTPases interact with various downstream effectors to induce cellular responses.

RhoB, together with the closely related RhoA and RhoC, form the Rho subfamily within the Rho GTPase family. Despite the high sequence homology between

\footnotetext{
*Correspondence: fmvega@us.es; anne.ridley@kcl.ac.uk

${ }^{1}$ Randall Division of Cell and Molecular Biophysics, King's College London, New Hunt's House, Guy's Campus, London SE1 1UL, UK

${ }^{2}$ Current address: Instituto de Biomedicina de Sevilla, Hospital Universitario Virgen del Rocío/CSIC/Universidad de Sevilla, Edificio IBiS, E-14013 Seville, Spain
}

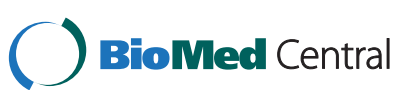

(C) 2015 Vega et al.; licensee BioMed Central. This is an Open Access article distributed under the terms of the Creative Commons Attribution License (http://creativecommons.org/licenses/by/4.0), which permits unrestricted use, distribution, and reproduction in any medium, provided the original work is properly credited. The Creative Commons Public Domain Dedication waiver (http://creativecommons.org/publicdomain/zero/1.0/) applies to the data made available in this article unless otherwise stated. these three proteins, RhoB has distinct biochemical and biological properties compared with RhoA and RhoC. RhoA and RhoC are modified at their C-terminus by the addition of a geranylgeranyl group, whereas RhoB can also be farnesylated. RhoB is the only Rho subfamily member that can be modified by palmitoylation [2,3]. RhoA and RhoC interact with RhoGDI, which extracts them from membranes by binding to the geranylgeranyl group and they are mostly localized in the cytoplasm. On the other hand, RhoB localizes mostly on the plasma membrane and/or on endosomes and does not bind to RhoGDI $[4,5]$. Consistent with its endosomal localization, RhoB regulates the trafficking of growth factor tyrosine kinase receptors through endosomes, including EGF receptor and VEGF receptor, and of the non-receptor tyrosine kinase Src, to the plasma membrane [6,7]. RhoB has also been described to localize to cell-cell junctions between Sertoli cells and germ cells in the testis [8].

RhoB has been postulated to act as a tumor suppressor in cancer and regulate apoptosis [9]. RhoB expression is reduced in several tumor types, including some prostate carcinomas, compared to non-cancer tissues and it is

ras otherwise stated. 
targeted by the miRNA miR21, involved in cancer progression $[10,11]$. RhoB expression is also induced by a variety of stresses including DNA damage, via JNK-mediated transcriptional upregulation $[12,13]$. RhoB overexpression inhibits proliferation, migration and invasion of gastric carcinoma cells [14]. On the other hand, mouse macrophages lacking RhoB, or human PC3 prostate cancer cells depleted of RhoB by RNAi, migrate faster than control cells. This correlates with reduced $\beta$ integrin levels on the cell surface $[15,16]$.

Epithelial cell-cell junction disruption occurs during progression of epithelial cancers [17]. E-cadherin is a homotypic cell-cell adhesion receptor that forms adherens junctions in epithelial cells and its localization to cell-cell contacts is dynamically regulated to control epithelial integrity during development and cancer progression [18].

Here we describe a new function for RhoB in maintaining cell-cell junctions in epithelial DU145 prostate cancer cells by regulating E-cadherin expression and localization. We also show that RhoB controls the levels of N-cadherin the mesenchymal-like PC3 prostate cancer cell line, which does not express E-cadherin. Decreased RhoB expression increases the migration of DU145 cells following the reduction in cell-cell adhesion.

\section{Results and discussion}

RhoB regulates cell-cell adhesion in epithelial cells

We have previously shown that RhoB depletion by RNAi alters focal adhesions and reduces $\beta 1$ integrin levels in PC3 prostate cancer cells. This correlates with increased migration speed of cells that predominantly move as single cells, such as macrophages and PC3 prostate cancer cells $[15,16,19]$. In addition to the plasma membrane and endosomes, RhoB has been reported to localize to cell-cell adhesions in some models [8]. To investigate whether RhoB could regulate cell-cell adhesions between epithelial cells, we depleted RhoB by RNAi in DU145 prostate cancer cells, which are epithelial in morphology and form colonies with E-cadherin-based adherens junctions $[20,21]$. siRNAs for RhoB were extensively used and characterized in our previous papers $[15,22]$ and no change in RhoA or RhoC activity was observed after RhoB depletion. RhoB-depleted DU145 cells were less able to form colonies than control cells (Figure 1A and B). We quantified the effects of RhoB on cell-cell adhesion by performing a neighbor analysis that quantifies the average number of cells touching each individual cell in the image (neighbors). RhoB-depleted DU145 cells had a significant reduction in the number of cells forming cell-cell contacts with each other (Figure $1 C$ and D). This change was accompanied by an increase in migration speed in the cell population (Figure 1E), mostly due to an increase in the migration of single cells not forming cell- cell contacts (Additional file 1). These changes were not due to a decrease in cell number as we have previously shown that RhoB knockdown does not affect cell proliferation [15]. Our previous analysis of PC3 cell morphology suggested that the RhoGEF GEF-H1 could act upstream to RhoB [15]. Interestingly, depletion of GEF-H1 also reduced cell-cell interaction (Figure 1B-D), suggesting that it plays a role in cell-cell interaction, potentially by regulating RhoB activity.

\section{RhoB alters E-cadherin expression and junctional maturation}

Because RhoB depletion reduced cell-cell interaction, we analyzed its effect on adherens junction components. RhoB depletion by RNAi reduced the expression of Ecadherin, a major component of adherens junctions, in DU145 cells (Figure 2A-C). No significant E-cadherin mRNA reduction was observed indicating that RhoB knockdown reduced E-cadherin expression at the protein level. We also observed a decrease in the levels of $\beta$-catenin, a cytoplasmic protein that interacts directly with E-cadherin [23], although we did not see an accumulation of $\beta$-catenin in the nucleus after RhoB knockdown. Interestingly endogenous RhoB could be partially localized to cell-cell adhesions in DU145 prostate epithelial cells, where it co-localized with E-cadherin (Figure 2D). This is consistent with our observation that RhoB regulates E-cadherin levels. The decrease in E-cadherin expression after RhoB depletion would be predicted to alter cell-cell junction organization. Together with the decrease in E-cadherin, we observed more disorganized ß-catenin localization at cell-cell contacts in RhoBdepleted cells that still conserved cell-cell adhesions (Figure 2E). $\beta$-catenin showed a 'zipper' distribution as described for early immature adherens junctions $[24,25]$. RhoB depletion also seemed to alter actin filament distribution: cells within colonies had reduced cortical actin filaments and instead had oriented stress fibers which appeared to connect to the discontinuous 'zipper-like' adherens junctions as described previously for endothelial cells [24]. At a later stage stress fibers are probably lost as observed most clearly in detached or more loosely adhered cells. These changes in stress fibers were not due to changes in RhoA activity after RhoB knockdown ([22] and data not shown) but could be a consequence of changes in cell-cell contact mediated tension. Similarly, ZO-1, a component of tight junctions [26], also showed 'zipper' localization in RhoB-depleted DU145 cells (Figure 3 ).

We propose that the effect of RhoB on cadherins could contribute to its tumor suppression function by participating in maintaining epithelial integrity. Reduced cadherin expression, due to a decrease in RhoB expression, increases migration in epithelial cancer cells. 


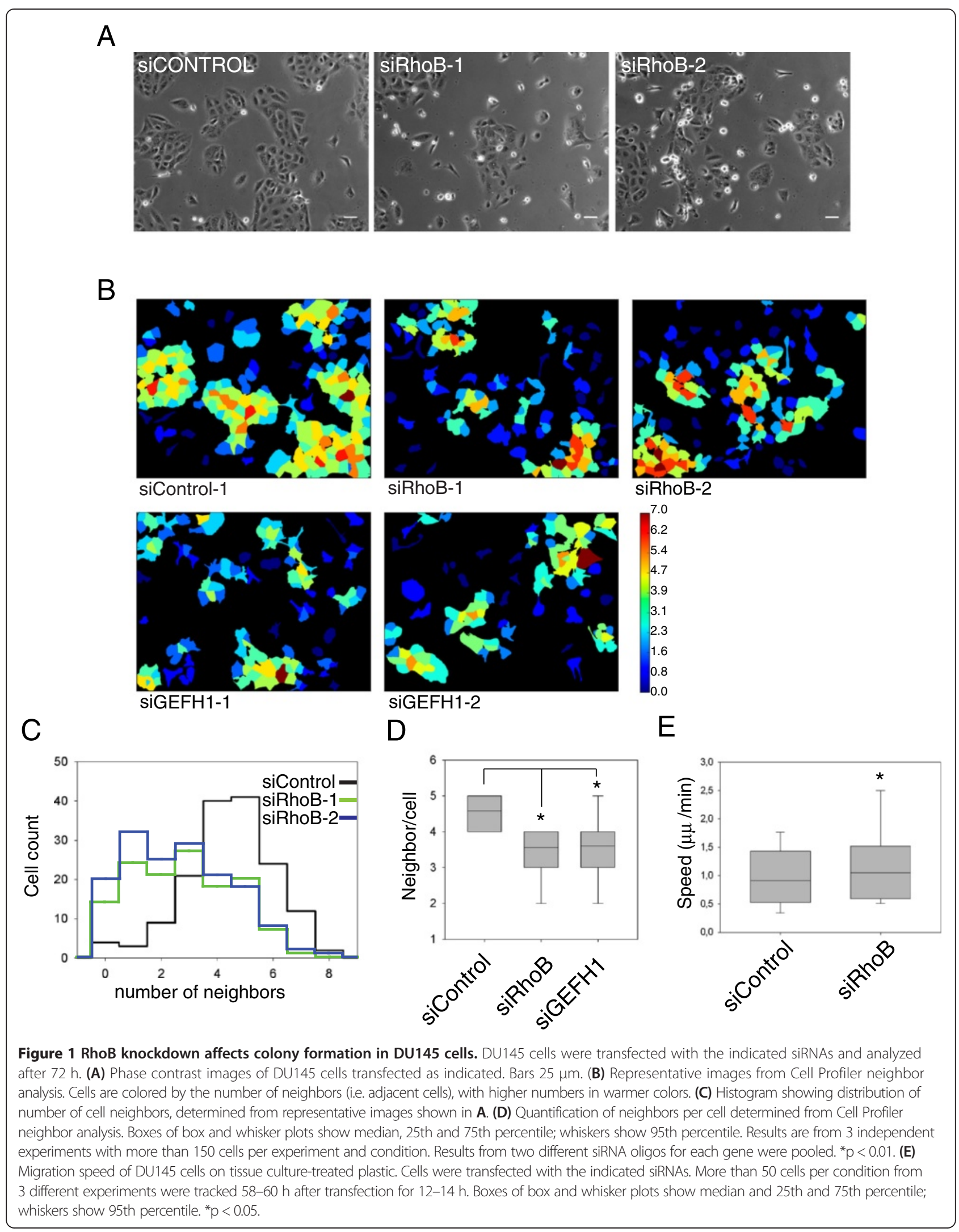




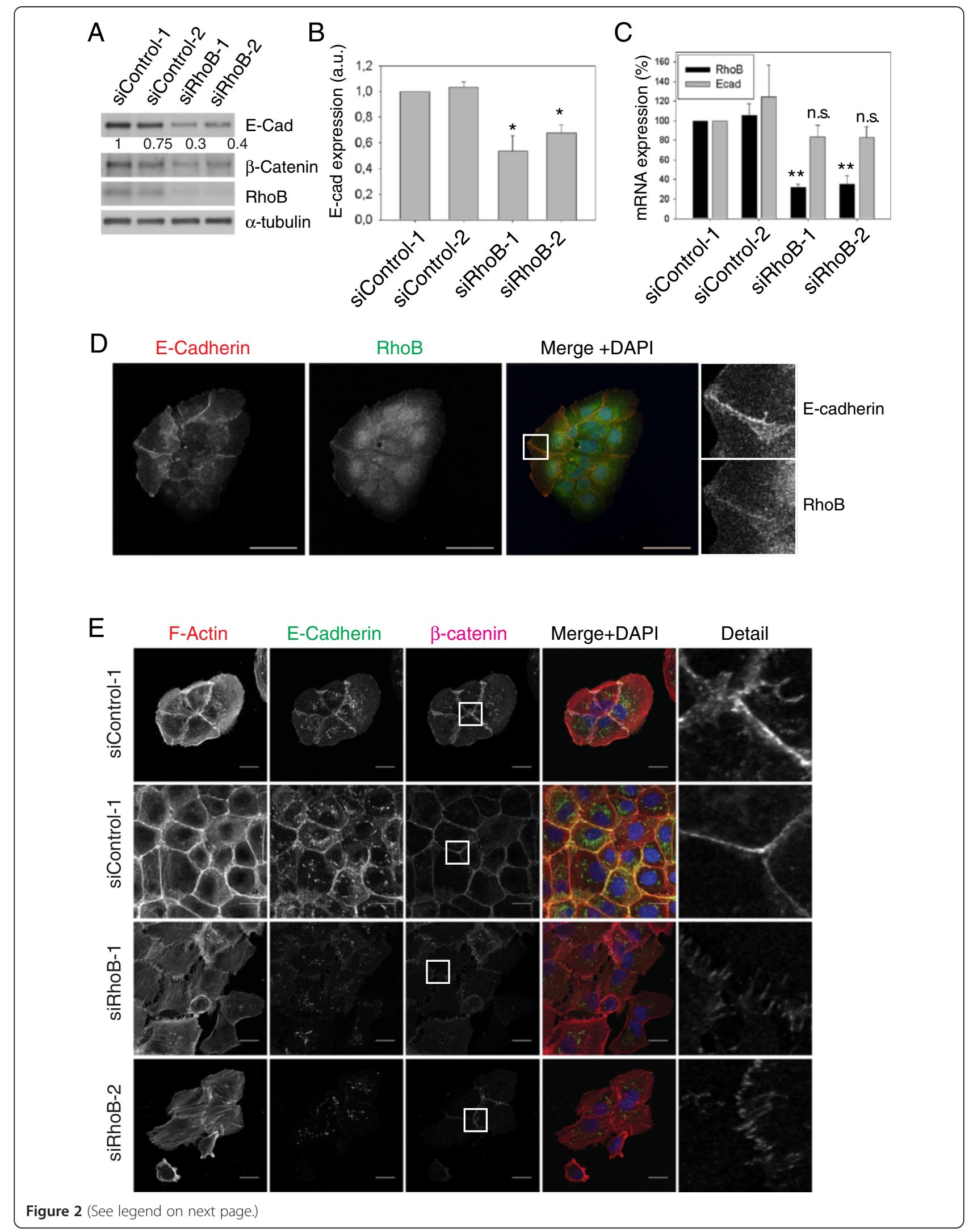


(See figure on previous page.)

Figure 2 RhoB regulates E-cadherin levels and cell-cell junction integrity. (A) Western blot of lysates from DU145 cells transfected with the indicated siRNAs and probed with the indicated antibodies. Relative levels of E-cadherin expression normalized to a-tubulin loading control are shown below the blot. (B) Quantification of E-cadherin expression from western blots. Graph shows mean expression of E-cadherin +/- s.e.m. relative to siControl-1 from 4 different experiments; ${ }^{*} \mathrm{p}<0.05$. a.u. arbitrary units. (C) Results from RT-PCR showing mean relative mRNA expression (+/- s.d.; $n=3)$. **p < 0.005. n.s., not significant. (D) Immunofluorescence showing RhoB and E-cadherin staining in DU145 cells. Images on right show magnified images of the box marked in the merge panels. Bars $50 \mu \mathrm{m}$. (E) DU145 cells transfected with the indicated siRNAs were stained for F-actin and nuclei (DAPI in merge images) and with antibodies to E-cadherin and $\beta$-catenin. Detail panels show magnified images from the boxed regions in the $\beta$-catenin images. Bars $20 \mu \mathrm{m}$.

\section{RhoB downregulation induces E-cadherin endosomal accumulation}

E-cadherin endocytosis and recycling is important for cell-cell junction formation, turnover and maintenance [18]. RhoB can regulate endosomal trafficking and recycling of membrane receptors [6,7]. After RhoB depletion, the remaining E-cadherin accumulated in EEA1-positive vesicles, a marker for early endosomes (Figure 4A) indicating a problem in the recycling of E-cadherin from endosomes to the plasma membrane. Although RhoB can also localize to lysosomes [27], we did not observe co-localization of E-cadherin with the lysosomal marker LAMP1 (Figure 4B). These results indicate that RhoB contributes to cell-cell junction integrity in epithelial cells by regulating both E-cadherin expression and localization. It is likely that altered traffic of cadherins through endosomes by RhoB depletion ultimately increases cadherin degradation by targeting them to multivesicular bodies [28]. We did not observe significant cadherin localization in lysosomes, possibly because it had already largely been degraded by the time multivesicular bodies fused with lysosomes.

\section{RhoB regulates $\mathrm{N}$-cadherin expression in PC3 cells}

We have previously reported that RhoB regulates migration in PC3 prostate cancer cells by controlling focal adhesion dynamics and $\beta 1$ integrin levels and localization
[15]. N-cadherin expression levels can modulate cell migration and polarity by affecting integrin-based adhesions in tumor astrocytes [29]. As we have shown that RhoB regulates E-cadherin levels in epithelial cells, we investigated whether RhoB affects $\mathrm{N}$-cadherin levels in cells where E-cadherin is not present. In control PC3 cells, which have undetectable levels of E-cadherin (data not shown), N-cadherin localized to actin-rich protrusions and to transient regions of cell-cell contact (Figure 5A), which are not very frequent in this mesenchymal cell line, as previously described [30]. RhoB depletion reduced $\mathrm{N}$-cadherin levels in PC3 cells, and cells were rarely observed to interact with each other (Figure 5A-C). Consistent with this, timelapse movies indicated that RhoB-depleted PC3 cells formed fewer stable contacts with other cells when they touched (Additional file 2), which could reflect the reduced N-cadherin levels. However, N-cadherin depletion in PC3 cells did not induce the same morphological phenotype as RhoB depletion (data not shown), and thus it is unlikely that the effect of RhoB on cell shape [15] is due solely to altered $\mathrm{N}$-cadherin levels. Integrin depletion and reduced adhesion to the substratum is probably the major mechanism underlying the observed phenotype [15] but $\mathrm{N}$-cadherin downregulation could contribute to the increase in migration speed by reducing PC3 cell-cell adhesion.

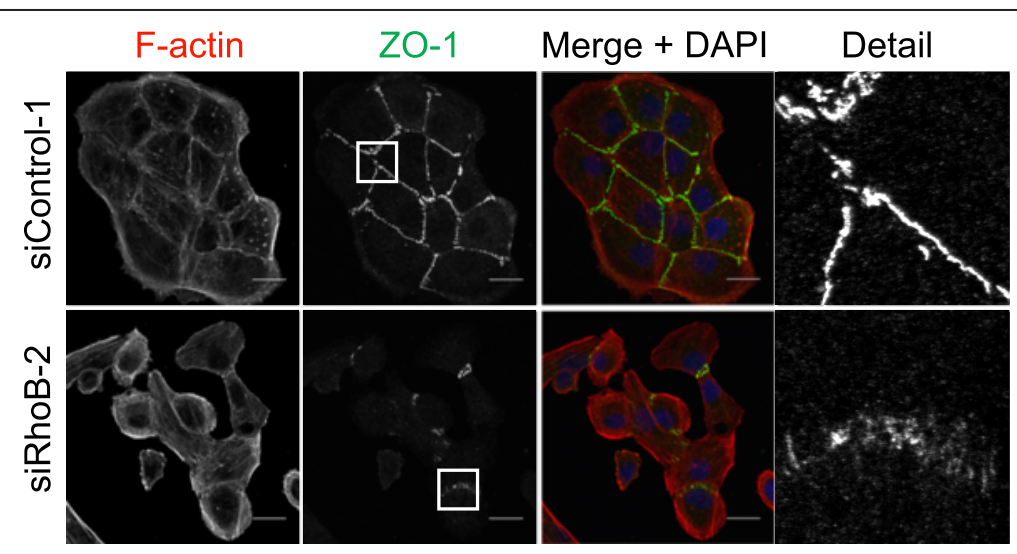

Figure 3 RhoB affects cell-cell junctions. DU145 cells transfected with the indicated siRNAs were stained for F-actin and nuclei (DAPI in merge) and with antibodies to ZO-1. Detail panels show magnified images from the boxed regions in the ZO-1 images. Bars $20 \mu \mathrm{m}$. 


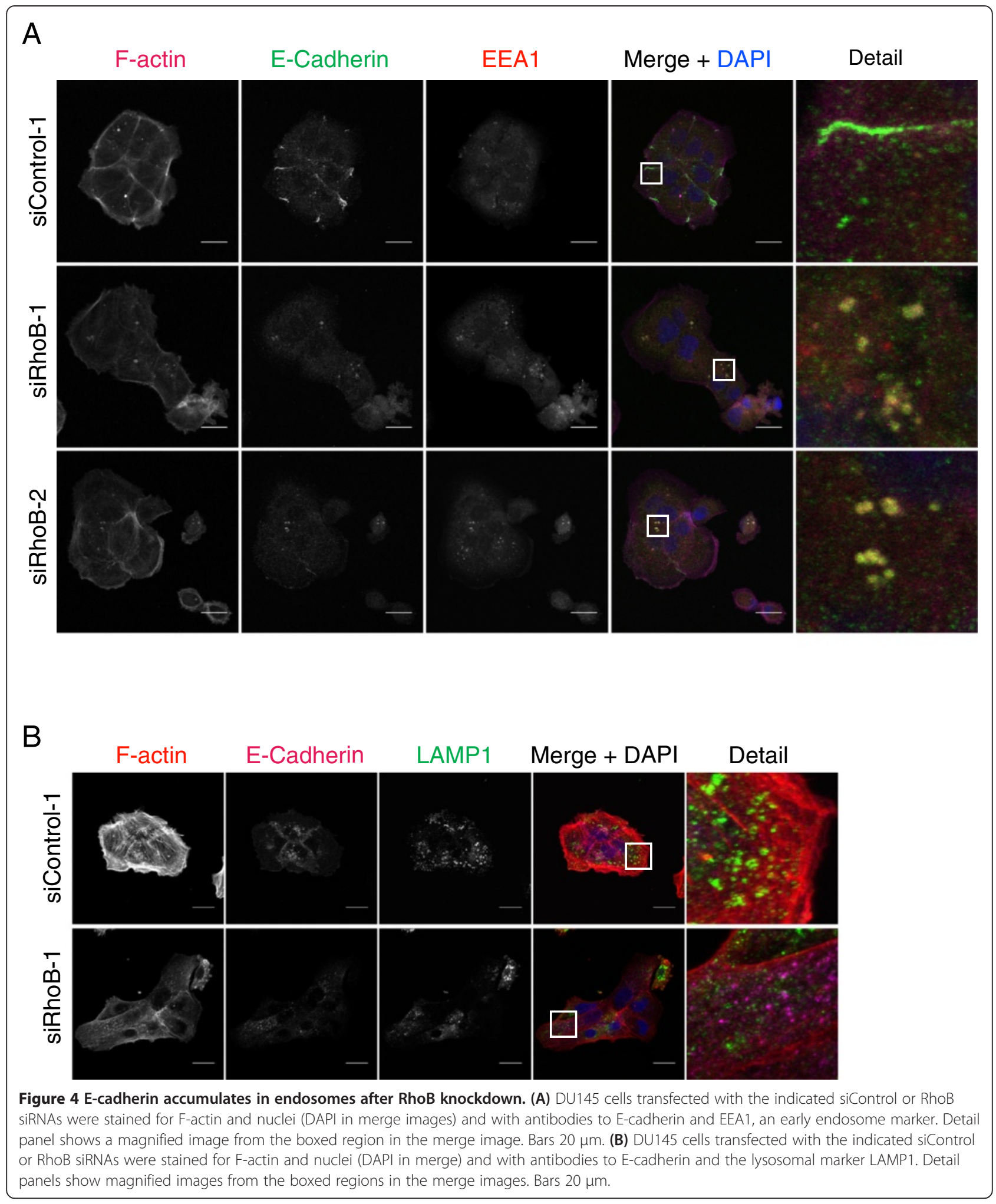

Taken together, these results indicate that RhoB affects the expression and localization of cadherins and thereby alters cell-cell adhesion. RhoB has previously been described to regulate surface levels of EGF receptor and
VEGF receptor and integrins $[6,7,15,19]$. Our work extends the types of membrane receptors regulated by RhoB to include cadherins. RhoB is often downregulated in prostate carcinomas compared with normal prostate 

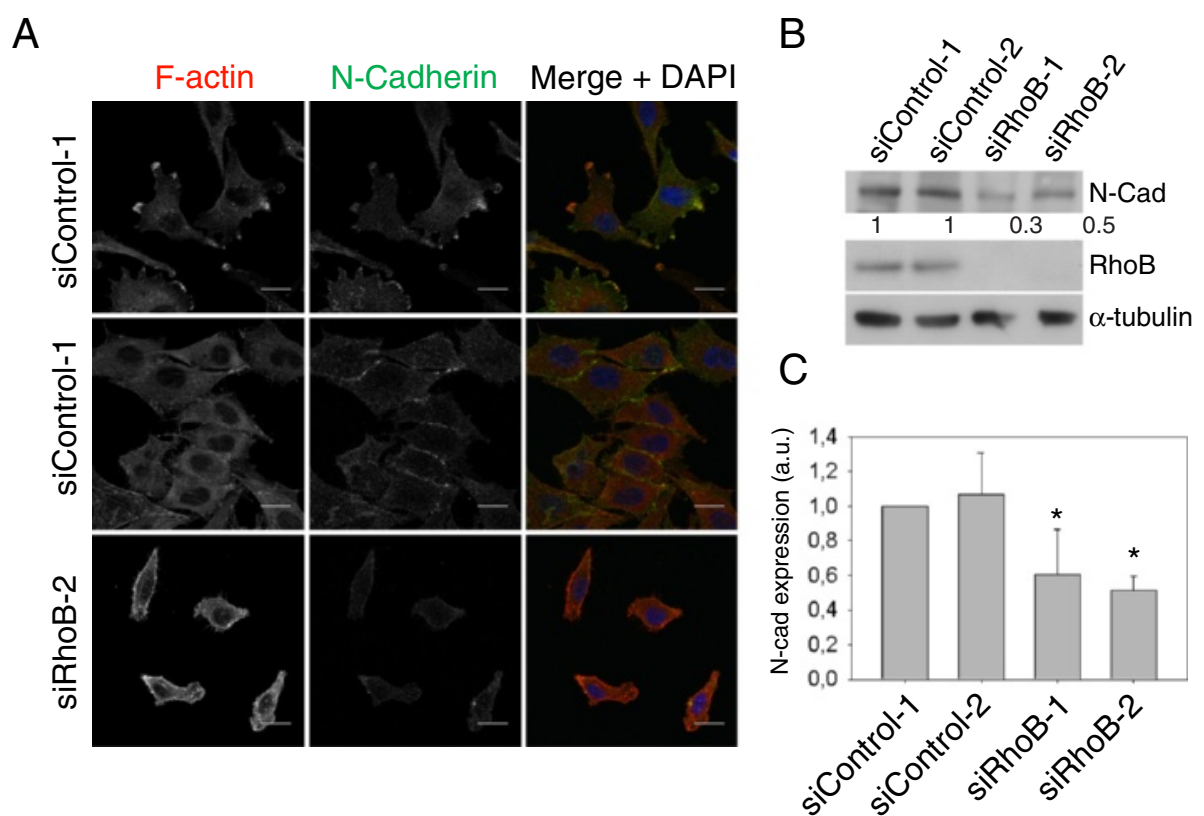

Figure 5 RhoB reduces $\mathbf{N}$-cadherin levels in PC3 cells. (A) PC3 cells transfected with the indicated siRNAs were stained for F-actin and nuclei (DAPI in merge images), and with antibodies to N-cadherin. Bars $20 \mu \mathrm{m}$. (B) Western blot of cell lysates from PC3 cells transfected with the indicated siRNAs, and probed with the indicated antibodies. Relative levels of $\mathrm{N}$-cadherin expression normalized to a-tubulin loading control are shown below the blot. (C) Quantification of N-cadherin expression. Graph shows mean expression of N-cadherin relative to siControl-1+/- s.e.m. from 4 different experiments; ${ }^{*}<<0.05$. a.u., arbitrary units.

tissues (Oncomine; www.oncomine.org). Although RhoB knockout mice do not have a clear defect in epithelial cell interactions [31], they have not been tested in models where increased junctional turnover would be revealed, for example in epithelial cancer progression models. RhoB knockout mice nevertheless are more susceptible to carcinogen-induced skin tumors [31] although the possible involvement of E-cadherin downregulation has not been tested in this model. We propose that the effect of RhoB on cadherins could contribute to its tumor suppressor function by participating in maintaining epithelial integrity. Reduced cadherin expression, due to a decrease in RhoB expression, increases migration in epithelial cancer cells. In epithelial-derived cancer cells that have lost or lack Ecadherin expression, decreased RhoB could also promote migration by its effect on integrins [15].

\section{Conclusions}

The results presented here show that RhoB can affect levels and localization of cadherins in prostate cancer cells, thus participating in the maintenance of epithelial integrity. Reduced cadherin levels induced by RhoB downregulation lead to increased migration of cells that normally have an epithelial morphology with stable cellcell junctions. In cells with epithelial origin which have lost E-cadherin expression but express $\mathrm{N}$-cadherin, its regulation by RhoB could also contribute to a more migratory phenotype. Our work extends the types of membrane receptors regulated by RhoB to include cadherins and indicates a mechanism by which RhoB downregulation can contribute to tumor progression in prostate cancer.

\section{Methods}

\section{Cell lines and reagents}

DU145 and PC3 prostate cancer cell lines were grown in RPMI containing $25 \mathrm{mM}$ Hepes and $2 \mathrm{mM}$ glutamine supplemented with $10 \% \mathrm{FCS}, 100 \mu \mathrm{g} / \mathrm{ml}$ streptomycin and 100 units $/ \mathrm{ml}$ penicillin. The following antibodies were used: rabbit polyclonal (Santa Cruz 119, sc-180) or mouse monoclonal (Cell Signaling) RhoB antibodies, $\alpha$-tubulin DM1A clone (Sigma, T6199), mouse monoclonal (BD Transduction, 610181) or rabbit polyclonal (Cell Signaling) E-cadherin, N-cadherin (BD Transduction), ZO-1 (Invitrogene), EEA1 (Santa Cruz), LAMP1 (Santa Cruz) and $\beta$-catenin (BD Transduction, 610153). Secondary HRP-labelled antibodies were from Amersham. Protease inhibitor cocktail (complete) was from Roche and Phosphatase inhibitor cocktail II and IV from Calbiochem.

\section{Transfections and western blotting}

All siRNAs were from Dharmacon (GE Healthcare): siRhoB-1 (CAUCCAAGCCUACGACUAC), siRhoB-2 (GCAUCCAAGCCUACGACUA), on target plus siControl non-targeting siRNAs 1 and 2 (D-001810-01, D-001810-02), 
GEFH1-1 (CAACAUUGCUGGACAUUUC), GEFH1-2 (GAAUUAAGAUGGAGUUGCA). All siRNAs were initially tested for knockdown of the relevant protein in cells. Cell transfection, lysate preparation and immunoblotting were performed as described previously [22]. Cell lysates for western blot analysis were prepared $72 \mathrm{~h}$ after siRNA transfection.

\section{Timelapse microscopy}

For phase contrast timelapse microscopy, a fully motorized, multi-field Nikon TE2000 microscope was used. Cell migration experiments were carried out as described [22]. Unless indicated, cells were imaged in medium containing $1 \%$ FCS.

\section{Confocal microscopy and cell shape and neighbor analysis} Immunofluorescence staining was carried out as previously described [22]. Alexa Fluor phalloidin (wavelengths $480 \mathrm{~nm}, 543 \mathrm{~nm}$ or $633 \mathrm{~nm}$, Molecular Probes) was used for F-actin visualization, DAPI for nuclear staining, FITClabelled $\alpha$-tubulin antibody (DM1A clone) for microtubule staining and primary antibodies followed by Alexa Fluorconjugated secondary antibodies (Molecular Probes) for ZO-1, $\beta$-catenin, E-cadherin, RhoB, LMP-1 or EEA1 stainings. Confocal images were acquired with a Zeiss LSM510 inverted confocal microscope. Morphology analysis was carried out using Metamorph or Cell Profiler software [32] from F-actin-stained fluorescence images. For neighbor analysis DU145 cells were transfected with siRNA and seeded at subconfluence. Cultures were left to grow for $72 \mathrm{~h}$ before fixing and staining for F-actin and DAPI. Neighbor analysis was performed with Cell Profiler [32] from fluorescence images.

\section{RT-PCR}

Total RNA was extracted from DU145 cells $50 \mathrm{~h}$ post siRNA transfection (RNeasy MICRO kit; Qiagen) and reverse transcribed with the QuantiTec Reverse transcription kit (Qiagen) according to the manufacturer's instructions. Quantitative real time PCR was performed with $20 \mathrm{ng}$ of cDNA in a ViiA7 thermocycler using SYBR Green detection system (Applied biosystems). Data analysis was performed with the $\Delta C t$ method. Specific oligonucleotides were used for the detection of Ecadherin (TCGACACCCGATTCAAAGTG, GTCCCAG GCGTAGACCAAGA) or RhoB (CCCACCGTCTTCGA GAACTA, CACCGAGAAGCACATGAGAA) and GAP DH oligonucleotides (GTGAAGGTCGGAGTCAACG, TGAGGTCAATGAAGGGGTC) were used for loading control.

\section{Statistical analysis}

For statistical significance analysis either an unpaired twotailed t-test (for comparing two conditions) or a One-way
ANOVA with Dunn's Multiple Comparison post Test (for comparing multiple conditions) was used. All significances indicated are compared to siControl condition unless stated. At least 2 different siRNAs for each gene were analysed in every assay and results from the 2 siRNAs were pooled in some graphs.

\section{Additional files}

Additional file 1: DU145 cell migration. Timelapse movie of DU145 cells transfected with siControl-1 siRNA or siRhoB-2 siRNA. siRhoB transfected cultures have more single cells that migrate faster. Bar $50 \mu \mathrm{m}$.

Additional file 2: PC3 cell-cell contacts. Timelapse movie of PC3 cells transfected with siControl-1 siRNA or siRhoB-2 siRNA. siRhoB transfected cells are less prone to form stable cell-cell contacts when they touch than control cells. Bar $50 \mu \mathrm{m}$.

\section{Abbreviations}

GEF: Guanine nucleotide exchange factor; GAP: GTPase activating protein; RhoGDI: Rho GDP-dissociation inhibitor; JNK: Jun N-terminal kinase; FCS: Fetal calf serum; RT-PCR: Real time polymerase chain reaction.

\section{Competing interests}

The authors declare that they have no competing interests.

\section{Authors' contributions}

FMV and AJR conceived and designed the study and wrote the manuscript. FMV carried out most of the experiments. MT carried out DU145 migration analysis. NR participated in the design of the study and preliminary data. All authors read and approved the final manuscript.

\section{Acknowledgements}

We thank Dr. Ana Galán (Instituto de Biomedicina de Sevilla) for her help with the E-cadherin RT-PCR. This work was funded by the Breast Cancer Campaign, Cancer Research UK and Bettencourt-Schueller Foundation. Francisco M. Vega was supported in part by a Marie Curie fellowship (European Commission Project No. 24441).

Received: 14 November 2014 Accepted: 14 January 2015 Published online: 29 January 2015

\section{References}

1. Jaffe $A B$, Hall A. RHO GTPASES: biochemistry and biology. Annu Rev Cell Dev Biol. 2005;21:247-69.

2. Roberts PJ, Mitin N, Keller PJ, Chenette EJ, Madigan JP, Currin RO, et al. Rho Family GTPase modification and dependence on CAAX motif-signaled posttranslational modification. J Biol Chem. 2008;283:25150-63.

3. Adamson P, Marshall CJ, Hall A, Tilbrook PA. Post-translational modifications of p21rho proteins. J Biol Chem. 1992;267:20033-8.

4. Michaelson D, Silletti J, Murphy G, D’Eustachio P, Rush M, Philips MR. Differential localization of Rho GTPases in live cells: regulation by hypervariable regions and RhoGDI binding. J Cell Biol. 2001;152:111-26.

5. Adamson P, Paterson HF, Hall A. Intracellular localization of the P21rho proteins. J Cell Biol. 1992;119:617-27.

6. Fernandez-Borja M, Janssen L, Verwoerd D, Hordijk P, Neefjes J. RhoB regulates endosome transport by promoting actin assembly on endosomal membranes through Dia1. J Cell Sci. 2005;118:2661-70.

7. Sandilands E, Cans C, Fincham VJ, Brunton VG, Mellor H, Prendergast GC, et al. RhoB and actin polymerization coordinate Src activation with endosome-mediated delivery to the membrane. Dev Cell. 2004;7:855-69.

8. Lui WY, Lee WM, Cheng CY. Sertoli-germ cell adherens junction dynamics in the testis are regulated by RhoB GTPase via the ROCK/LIMK signaling pathway. Biol Reprod. 2003;68:2189-206.

9. Huang M, Prendergast GC. RhoB in cancer suppression. Histol Histopathol. 2006;21:213-8 
10. Liu M, Tang Q, Qiu M, Lang N, Li M, Zheng Y, et al. miR-21 targets the tumor suppressor RhoB and regulates proliferation, invasion and apoptosis in colorectal cancer cells. FEBS Lett. 2011;585:2998-3005.

11. Connolly EC, Van Doorslaer K, Rogler LE, Rogler CE. Overexpression of miR-21 promotes an in vitro metastatic phenotype by targeting the tumor suppressor RHOB. Mol Cancer Res. 2010;8:691-700.

12. Kim BK, Kim HM, Chung KS, Kim DM, Park SK, Song A, et al. Upregulation of RhoB via C-Jun $\mathrm{N}$-terminal kinase signaling induces apoptosis of the human gastric carcinoma NUGC-3 cells treated with NSC12618. Carcinogenesis. 2011;32:254-61.

13. Kim DM, Won M, Chung CS, Kim S, Yim HJ, Jung SH, et al. JNK-mediated transcriptional upregulation of RhoB is critical for apoptosis of HCT-116 colon cancer cells by a novel diarylsulfonylurea derivative. Apoptosis. 2010;15:1540-8.

14. Zhou J, Zhu Y, Zhang G, Liu N, Sun L, Liu M, et al. A distinct role of RhoB in gastric cancer suppression. Int J Cancer. 2011;128:1057-68.

15. Vega FM, Colomba A, Reymond N, Thomas M, Ridley AJ. RhoB regulates cell migration through altered focal adhesion dynamics. Open Biol. 2012;2:120076.

16. Wheeler AP, Ridley AJ. RhoB affects macrophage adhesion, integrin expression and migration. Exp Cell Res. 2007;313:3505-16.

17. Le Bras GF, Taubenslag KJ, Andl CD. The regulation of cell-cell adhesion during epithelial-mesenchymal transition, motility and tumor progression. Cell Adhes Migr. 2012;6:365-73.

18. Baum B, Georgiou M. Dynamics of adherens junctions in epithelial establishment, maintenance, and remodeling. J Cell Biol. 2011;192:907-17.

19. Alfano D, Ragno P, Stoppelli MP, Ridley AJ. RhoB regulates UPAR signalling. J Cell Sci. 2012:125:2369-80.

20. Wells CM, Ahmed T, Masters JR, Jones GE. Rho family GTPases are activated during HGF-stimulated prostate cancer-cell scattering. Cell Motil Cytoskeleton. 2005;62:180-94.

21. Kumper S, Ridley AJ. p120ctn and P-cadherin but not E-cadherin regulate cell motility and invasion of DU145 prostate cancer cells. PLoS One. 2010;5: e11801.

22. Vega FM, Fruhwirth $\mathrm{G}, \mathrm{Ng}$ T, Ridley AJ. RhoA and RhoC have distinct roles in migration and invasion by acting through different targets. J Cell Biol. 2011;193:655-65.

23. Harris TJ, Tepass U. Adherens junctions: from molecules to morphogenesis. Nat Rev Mol Cell Biol. 2010;11:502-14

24. Millan J, Cain RJ, Reglero-Real N, Bigarella C, Marcos-Ramiro B, FernandezMartin $L$, et al. Adherens junctions connect stress fibres between adjacent endothelial cells. BMC Biol. 2010:8:11.

25. Oas RG, Nanes BA, Esimai CC, Vincent PA, Garcia AJ, Kowalczyk AP p120-catenin and beta-catenin differentially regulate cadherin adhesive function. Mol Biol Cell. 2013;24:704-14.

26. Fanning AS, Anderson JM. Zonula occludens-1 and -2 are cytosolic scaffolds that regulate the assembly of cellular junctions. Ann N Y Acad Sci. 2009:1165:113-20.

27. Perez-Sala D, Boya P, Ramos I, Herrera M, Stamatakis K. The C-terminal sequence of RhoB directs protein degradation through an endo-lysosomal pathway. PLoS One. 2009;4:e8117.

28. Katzmann DJ, Odorizzi G, Emr SD. Receptor downregulation and multivesicular-body sorting. Nat Rev Mol Cell Biol. 2002;3:893-905.

29. Camand E, Peglion F, Osmani N, Sanson M, Etienne-Manneville S. $\mathrm{N}$-cadherin expression level modulates integrin-mediated polarity and strongly impacts on the speed and directionality of glial cell migration. J Cell Sci. 2012;125:844-57.

30. Valderrama F, Thevapala S, Ridley AJ. Radixin regulates cell migration and cell-cell adhesion through Rac1. J Cell Sci. 2012;125:3310-9.

31. Liu AX, Rane N, Liu JP, Prendergast GC. RhoB is dispensable for mouse development, but it modifies susceptibility to tumor formation as well as cell adhesion and growth factor signaling in transformed cells. Mol Cell Biol. 2001;21:6906-12.

32. Carpenter $A E$, Jones $T R$, Lamprecht MR, Clarke C, Kang $\mathbb{H}$, Friman $\mathrm{O}$, et al. Cell Profiler: image analysis software for identifying and quantifying cell phenotypes. Genome Biol. 2006;7:R100.

\section{Submit your next manuscript to BioMed Central and take full advantage of:}

- Convenient online submission

- Thorough peer review

- No space constraints or color figure charges

- Immediate publication on acceptance

- Inclusion in PubMed, CAS, Scopus and Google Scholar

- Research which is freely available for redistribution

Submit your manuscript at www.biomedcentral.com/submit 